\title{
A new explicit formula for the Bernoulli numbers in terms of the Stirling numbers of the second kind
}

\author{
Sumit Kumar Jha \\ International Institute of Information Technology \\ Hyderabad-500 032, India \\ e-mail: kumarjha.sumiteresearch.iiit.ac.in
}

Received: 15 January 2020

Abstract: Let $B_{r}$ denote the Bernoulli numbers, and $S(r, k)$ denote the Stirling numbers of the second kind. We prove the following explicit formula

$$
B_{r+1}=\sum_{k=0}^{r} \frac{(-1)^{k-1} k ! S(r, k)}{(k+1)(k+2)} .
$$

To the best of our knowledge, the formula is new.

Keywords: Bernoulli numbers, Stirling numbers of the second kind, Riemann zeta function, Polylogarithm function.

2010 Mathematics Subject Classification: 11B68.

\section{Introduction}

Definition 1.1. The Bernoulli numbers $B_{n}$ can be defined by the following generating function:

$$
\frac{t}{e^{t}-1}=\sum_{n \geq 0} \frac{B_{n} t^{n}}{n !}
$$

where $|t|<2 \pi$.

Definition 1.2. The Stirling number of the second kind, denoted by $S(n, m)$, is the number of ways of partitioning a set of $n$ elements into $m$ nonempty sets. 
There are many explicit formulas known for the Bernoulli numbers $[1,3,5-10,13,14]$. For example, all of the formulas below express the Bernoulli numbers explicitly in terms of the Stirling numbers of the second kind:

$$
\begin{aligned}
B_{r} & =\sum_{k=0}^{r} \frac{(-1)^{k} k ! S(r, k)}{k+1}, \\
B_{r} & =(-1)^{r} \sum_{k=1}^{r} \frac{(-1)^{k-1}(k-1) ! S(r, k)}{k+1}, \\
B_{r} & =\frac{r}{1-2^{r}} \sum_{k=1}^{r-1} \frac{(-1)^{k} k ! S(r-1, k)}{2^{k+1}}, \\
B_{r+1} & =\frac{(-1)^{r}(r+1) 2^{r-1}}{2^{r+1}-1} \sum_{k=1}^{r} \frac{(-1)^{k} k ! S(r, k)}{k+1} 2^{-2 k}\left(\begin{array}{c}
2 k \\
k
\end{array}\right), \\
B_{r} & =\sum_{i=0}^{r}(-1)^{i} \frac{\left(\begin{array}{c}
r+1 \\
i+1
\end{array}\right)}{\left(\begin{array}{c}
r+i \\
i
\end{array}\right)} S(r+i, i), \\
B_{r+1} & =-\frac{r+1}{4\left(1+2^{-(r+1)}\left(1-2^{-r}\right)\right)}\left(\sum_{k=0}^{r} \frac{(-1)^{k} S(r, k)}{k+1}\left(\frac{3}{4}\right)^{(k)}+4^{-r} E_{r}\right)
\end{aligned}
$$

where $x^{(n)}=x(x+1)(x+2) \cdots(x+n-1)$ denotes the rising factorial, and $\left(E_{r}\right)$ denotes the Euler numbers defined by the following generating function:

$$
\frac{1}{\cosh t}=\frac{2}{e^{t}+e^{-t}}=\sum_{n=0}^{\infty} \frac{E_{n}}{n !} t^{n} .
$$

The formula (1) has been generalized in $[4,11]$. In the following section, we derive a new explicit formula for the Bernoulli numbers in terms of the Stirling numbers of the second kind.

\section{Main result}

Our main result is the following.

Theorem 2.1. Let $r$ be any non-negative integer. Then we have

$$
B_{r+1}=\sum_{k=0}^{r} \frac{(-1)^{k-1} k ! S(r, k)}{(k+1)(k+2)} .
$$

Proof. We begin with the following result [2, formula 3.2.1.6]

$$
(s-1) \zeta(s)=-\int_{0}^{\infty} \frac{\operatorname{Li}_{s}(-x)}{(1+x)^{2}} d x,
$$

where $\zeta(s)$ is the Riemann zeta function, and $\operatorname{Li}_{s}(-x)$ is the polylogarithm function.

Letting $s=-r$, a negative integer, in the equation (2) we arrive at

$$
\int_{0}^{\infty} \frac{\operatorname{Li}_{-r}(-x)}{(1+x)^{2}} d x=(r+1) \zeta(-r)=-B_{r+1} .
$$


We use the following representation from the note [12]

$$
\mathrm{Li}_{-r}(-x)=\sum_{k=0}^{r} k ! S(r, k)\left(\frac{1}{1+x}\right)^{k+1}(-x)^{k}
$$

to conclude that

$$
\begin{array}{r}
B_{r+1}=-\int_{0}^{\infty} \frac{\mathrm{Li}_{-r}(-x)}{(1+x)^{2}} d x \\
=-\int_{0}^{\infty}\left(\sum_{k=0}^{r} \frac{(-1)^{k} k ! x^{k}}{(1+x)^{k+3}} S(r, k)\right) d x \\
=-\sum_{k=0}^{r} \frac{(-1)^{k} k ! S(r, k)}{(k+1)(k+2)} .
\end{array}
$$

This completes the proof.

\section{References}

[1] Fekih-Ahmed, L. (2012). On some explicit formulas for Bernoulli numbers and polynomials, preprint. Available at https://arxiv.org/abs/1106.5247.

[2] Brychkov, Y. A., Marichev, O. I., \& Savischenko, N. A. (2019). Handbook of Mellin Transforms, CRC Press, Boca Raton, FL.

[3] Gould, H. W. (1972). Explicit formulas for Bernoulli numbers, Amer. Math. Monthly, 79 (1), 44-51.

[4] Guo, B. N., Mező, I., \& Qi, F. (2016). An explicit formula for the Bernoulli polynomials in terms of the $r$-Stirling numbers of the second kind, Rocky Mountain J. Math., 46 (6), 1919-1923.

[5] Guo, B. N., \& Qi, F. (2014). Some identities and an explicit formula for Bernoulli and Stirling numbers, J. Comput. Appl. Math., 255, 568-579.

[6] Guo, B. N., \& Qi, F. (2015). An explicit formula for Bernoulli numbers in terms of Stirling numbers of the second kind, J. Anal. Number Theory 3 (1), 27-30.

[7] Jha, S. K. (2019). A congruence for the number of alternating permutations, preprint. Available at https://osf.io/yfz7h.

[8] Jha, S. K. (2019). A new explicit formula for Bernoulli numbers involving the Euler number, Mosc. J. Comb. Number Theory, 8 (4), 385-387.

[9] Jha, S. K. (2020). Two new explicit formulas for the Bernoulli numbers, Integers, 20, Article No. A21, 5 pp. 
[10] Jha, S. K. (2020). Two new explicit formulas for the even-indexed Bernoulli numbers, J. Integer Seq., 23 (2), Article No. 20.2.6.

[11] Jha, S. K. (2020). An explicit formula for the Bernoulli polynomials in terms of the Stirling numbers of the second kind, preprint. Available at https://osf.io/n832v.

[12] Landsburg, S. E. (2009). Stirling numbers and polylogarithms, preprint. Available at http://www. landsburg.com/query.pdf.

[13] Qi, F., \& Guo, B. N. (2014). Alternative proofs of a formula for Bernoulli numbers in terms of Stirling numbers, Analysis (Berlin), 34 (3), 311-317.

[14] Quaintance, J., \& Gould, H. W. (2015). Combinatorial Identities For Stirling Numbers: The Unpublished Notes of H. W. Gould, World Scientific. 